\title{
Levitin-Polyak Well-Posedness for Equilibrium Problems with Functional Constraints
}

\author{
Xian Jun Long, ${ }^{1}$ Nan-Jing Huang, ${ }^{1,2}$ and Kok Lay Teo ${ }^{3}$ \\ ${ }^{1}$ Department of Mathematics, Sichuan University, Chengdu, Sichuan 610064, China \\ ${ }^{2}$ State Key Laboratory of Oil and Gas Reservoir Geology and Exploitation, Chengdu 610500, China \\ ${ }^{3}$ Department of Mathematics and Statistics, Curtin University of Technology, \\ Perth W.A. 6102, Australia
}

Correspondence should be addressed to Nan-Jing Huang, nanjinghuang@hotmail.com

Received 8 November 2007; Accepted 11 December 2007

Recommended by Simeon Reich

We generalize the notions of Levitin-Polyak well-posedness to an equilibrium problem with both abstract and functional constraints. We introduce several types of (generalized) Levitin-Polyak well-posedness. Some metric characterizations and sufficient conditions for these types of wellposedness are obtained. Some relations among these types of well-posedness are also established under some suitable conditions.

Copyright ( 2008 Xian Jun Long et al. This is an open access article distributed under the Creative Commons Attribution License, which permits unrestricted use, distribution, and reproduction in any medium, provided the original work is properly cited.

\section{Introduction}

Equilibrium problem was first introduced by Blum and Oettli [1], which includes optimization problems, fixed point problems, variational inequality problems, and complementarity problems as special cases. In the past ten years, equilibrium problem has been extensively studied and generalized (see, e.g., $[2,3]$ ).

It is well known that the well-posedness is very important for both optimization theory and numerical methods of optimization problems, which guarantees that, for approximating solution sequences, there is a subsequence which converges to a solution. The wellposedness of unconstrained and constrained scalar optimization problems was first introduced and studied by Tykhonov [4] and Levitin and Polyak [5], respectively. Since then, various concepts of well-posedness have been introduced and extensively studied for scalar optimization problems [6-13], best approximation problems [14-16], vector optimization problems [17-23], optimization control problems [24], nonconvex constrained variational problems [25], variational inequality problems [26, 27], and Nash equilibrium problems [28-31]. The study 
of Levitin-Polyak well-posedness for convex scalar optimization problems with functional constraints started by Konsulova and Revalski [32]. Recently, Huang and Yang generalized those results to nonconvex (vector) optimization problems with both abstract and functional constraints [33, 34]. Very recently, Huang and Yang [35] studied Levitin-Polyak-type wellposedness for generalized variational inequality problems with abstract and functional constraints. They introduced several types of generalized Levitin-Polyak well-posednesses and obtained some criteria and characterizations for these types of well-posednesses.

Motivated and inspired by the numerical method introduced by Mastroeni [36] and the works mentioned above, the purpose of this paper is to generalize the results in [35] to equilibrium problems. We introduce several types of Levitin-Polyak well-posedness for equilibrium problems with abstract and functional constraints. Necessary and sufficient conditions for these types of well-posedness are obtained. Some relations among these types of wellposedness are also established under some suitable conditions.

\section{Preliminaries}

Let $(X,\|\cdot\|)$ be a normed space, and let $(Y, d)$ be a metric space. Let $K \subseteq X$ and $D \subseteq Y$ be nonempty and closed. Let $f$ from $X \times X$ to $R \cup\{ \pm \infty\}$ be a bifunction satisfying $f(x, x)=0$ for any $x \in X$ and let $g$ from $K$ to $Y$ be a function. Let $S=\{x \in K: g(x) \in D\}$.

In this paper, we consider the following explicit constrained equilibrium problem: finding a point $x \in S$ such that

$$
f(x, y) \geq 0, \quad \forall y \in S .
$$

Denote by $\Gamma$ the solution set of (EP). Throughout this paper, we always assume that $S \neq \varnothing$ and $g$ is continuous on $K$.

Let $(W, d)$ be a metric space and $W_{1} \subset W$. We denote by $d_{W_{1}}(p)=\inf \left\{d\left(p, p^{\prime}\right): p^{\prime} \in W_{1}\right\}$ the distance from the point $p$ to the set $W_{1}$.

Definition 2.1. A sequence $\left\{x_{n}\right\} \subset K$ is said to be as follows:

(i) type I Levitin-Polyak (LP in short) approximating solution sequence if there exists a sequence $\varepsilon_{n}>0$ with $\varepsilon_{n} \rightarrow 0$ such that

$$
\begin{gathered}
d_{S}\left(x_{n}\right) \leq \varepsilon_{n}, \\
f\left(x_{n}, y\right)+\varepsilon_{n} \geq 0, \quad \forall y \in S
\end{gathered}
$$

(ii) type II LP approximating solution sequence if there exists a sequence $\varepsilon_{n}>0$ with $\varepsilon_{n} \rightarrow 0$ and $\left\{y_{n}\right\} \subset S$ such that (2.1) and (2.2) hold, and

$$
f\left(x_{n}, y_{n}\right) \leq \varepsilon_{n}
$$

(iii) a generalized type I LP approximating solution sequence if there exists a sequence $\varepsilon_{n}>0$ with $\varepsilon_{n} \rightarrow 0$ such that

$$
d_{D}\left(g\left(x_{n}\right)\right) \leq \varepsilon_{n}
$$

and (2.2) hold; 
(iv) a generalized type II LP approximating solution sequence if there exists a sequence $\varepsilon_{n}>0$ with $\varepsilon_{n} \rightarrow 0$ and $\left\{y_{n}\right\} \subset S$ such that (2.2), (2.3), and (2.4) hold.

Definition 2.2. The explicit constrained equilibrium problem (EP) is said to be of type I (resp., type II, generalized type I, generalized type II) LP well-posed if the solution set $\Gamma$ of (EP) is nonempty, and for any type I (resp., type II, generalized type I, generalized type II) LP approximating solution sequence $\left\{x_{n}\right\}$ has a subsequence which converges to some point of $\Gamma$.

Remark 2.3. (i) If $f(x, y)=\langle F(x), y-x\rangle$ for all $x, y \in K$, where $F: K \rightarrow X^{*}$ is a mapping and $X^{*}$ denotes the topological dual of $X$, then type I (resp., type II, generalized type I, generalized type II) LP well-posedness for (EP) defined in Definition 2.2 reduces to type I (resp., type II, generalized type I, generalized type II) LP well-posedness for the variational inequality with functional constraints.

(ii) It is easy to see that any (generalized) type II LP approximating solution sequence is a (generalized) type I LP approximating solution sequence. Thus, (generalized) type I LP well-posedness implies (generalized) type II LP well-posedness.

(iii) Each type of LP well-posedness for (EP) implies that the solution set $\Gamma$ is nonempty and compact.

(iv) Let $g$ be a uniformly continuous function on the set

$$
S\left(\delta_{0}\right)=\left\{x \in K: d_{S}(x) \leq \delta_{0}\right\}
$$

for some $\delta_{0}>0$. Then, generalized type I (type II) LP well-posedness implies type I (type II) LP well-posedness.

It is well known that an equilibrium problem is closely related to a minimization problem (see, e.g., [36]). Thus, we need to recall some notions of LP well-posedness for the following general constrained optimization problem:

$$
\min h(x) \text { s.t. } x \in K, g(x) \in D \text {, }
$$

where $h: K \rightarrow R \cup\{+\infty\}$ is lower semicontinuous. The feasible set of $(\mathrm{P})$ is still denoted by $S$. The optimal set and optimal value of $(\mathrm{P})$ are denoted by $\bar{\Gamma}$ and $\bar{v}$, respectively. If $\operatorname{Dom}(h) \cap$ $S \neq \varnothing$, then $\bar{v}<+\infty$, where

$$
\operatorname{Dom}(h)=\{x \in K: h(x)<+\infty\} .
$$

In this paper, we always assume that $\bar{v}>-\infty$. In [33], Huang and Yang introduced the following LP well-posed for generalized constrained optimization problem $(\mathrm{P})$.

Definition 2.4. A sequence $\left\{x_{n}\right\} \subset K$ is said to be

(i) type I LP minimizing sequence for $(\mathrm{P})$ if

$$
\begin{gathered}
d_{S}\left(x_{n}\right) \longrightarrow 0, \\
\limsup _{n \rightarrow+\infty} h\left(x_{n}\right) \leq \bar{v} ;
\end{gathered}
$$


(ii) type II LP minimizing sequence for $(\mathrm{P})$ if

$$
\lim _{n \rightarrow+\infty} h\left(x_{n}\right)=\bar{v}
$$

and (2.7) holds;

(iii) a generalized type I LP minimizing sequence for $(\mathrm{P})$ if (2.8) holds and

$$
d_{D}\left(g\left(x_{n}\right)\right) \longrightarrow 0
$$

(iv) a generalized type II LP minimizing sequence for (P) if (2.9) and (2.10) hold.

Definition 2.5. The generalized constrained optimization problem (P) is said to be type I (resp., type II, generalized type I, generalized type II) LP well-posed if $\bar{v}$ is finite, $\bar{\Gamma} \neq \varnothing$ and for any type I (resp., type II, generalized type I, generalized type II) LP minimizing sequence $\left\{x_{n}\right\}$ has a subsequence which converges to some point of $\bar{\Gamma}$.

Mastroeni [36] introduced the following gap function for (EP):

$$
h(x)=\sup _{y \in S}\{-f(x, y)\}, \quad \forall x \in K
$$

It is clear that $h$ is a function from $K$ to $(-\infty,+\infty]$. Moreover, if $\Gamma \neq \varnothing$, then $\operatorname{Dom}(h) \cap S \neq \varnothing$.

Lemma 2.6 (see [36]). Let $h$ be defined by (2.11). Then

(i) $h(x) \geq 0$ for all $x \in S$;

(ii) $h(x)=0$ if and only if $x \in \Gamma$.

Remark 2.7. By Lemma 2.6, it is easy to see that $x_{0} \in \Gamma$ if and only if $x_{0}$ minimizes $h(x)$ over $S$ with $h\left(x_{0}\right)=0$.

Now, we show the following lemmas.

Lemma 2.8. Let $h$ be defined by (2.11). Suppose that $f$ is upper semicontinuous on $K \times K$ with respect to the first argument. Then $h$ is lower semicontinuous on $K$.

Proof. Let $\alpha \in R$ and let the sequence $\left\{x_{n}\right\} \subset K$ satisfy $x_{n} \rightarrow x_{0} \in K$ and $h\left(x_{n}\right) \leq \alpha$. It follows that, for any $\varepsilon>0$ and each $n,-f\left(x_{n}, y\right) \leq \alpha+\varepsilon$ for all $y \in S$. By the upper semicontinuity of $f$ with respect to the first argument, we know that $-f\left(x_{0}, y\right) \leq \alpha+\varepsilon$. This implies that $h\left(x_{0}\right) \leq \alpha+\varepsilon$. From the arbitrariness of $\varepsilon>0$, we have $h\left(x_{0}\right) \leq \alpha$ and so $h$ is lower semicontinuous on $K$. This completes the proof.

Remark 2.9. Lemma 2.8 implies that $h$ is lower semicontinuous. Therefore, if $\operatorname{Dom}(h) \cap S \neq \varnothing$, then it is easy to see that Theorems 2.1 and 2.2 of [33] are true.

Lemma 2.10. Let $\Gamma \neq \varnothing$. Then, (EP) is type I (resp., type II, generalized type I, generalized type II) LP well-posed if and only if $(P)$ is type I (resp., type II, generalized type I, generalized type II) LP well-posed with $h$ defined by (2.11). 
Proof. Since $\Gamma \neq \varnothing$, it follows from Lemma 2.6 that $x_{0}$ is a solution of (EP) if and only if $x_{0}$ is an optimal solution of $(\mathrm{P})$ with $\bar{v}=h\left(x_{0}\right)=0$, where $h$ is defined by (2.11). It is easy to check that a sequence $\left\{x_{n}\right\}$ is a type I (resp., type II, generalized type I, generalized type II) LP approximating solution sequence of (EP) if and only if it is a type I (resp., type II, generalized type I, generalized type II) LP minimizing sequence of (P). Thus, the conclusions of Lemma 2.10 hold. This completes the proof.

Consider the following statement:

$\{\Gamma \neq \varnothing$ and, for any type I (resp., type II, generalized type I, generalized type II)

LP approximating solution sequence $\left\{x_{n}\right\}$, we have $\left.d_{\Gamma}\left(x_{n}\right) \longrightarrow 0\right\}$.

It is easy to prove the following lemma by Definition 2.2.

Lemma 2.11. If $(E P)$ is type I (resp., type II, generalized type I, generalized type II) LP well-posed, then (2.12) holds. Conversely, if (2.12) holds and $\Gamma$ is compact, then (EP) is type I (resp., type II, generalized type I, generalized type II) LP well-posed.

\section{Metric characterizations of LP well-posedness for (EP)}

In this section, we give some metric characterizations of various types of LP well-posedness for (EP) defined in Section 2.

Given two nonempty subsets $A$ and $B$ of $X$, the Hausdorff distance between $A$ and $B$ is defined by

$$
H(A, B)=\max \{e(A, B), e(B, A)\},
$$

where $e(A, B)=\sup _{a \in A} d(a, B)$ with $d(a, B)=\inf _{b \in B} d(a, b)$. tively, by

For any $\varepsilon>0$, two types of the approximating solution sets for (EP) are defined, respec-

$$
\begin{gathered}
M_{1}(\varepsilon)=\left\{x \in K: f(x, y)+\varepsilon \geq 0, \forall y \in S, d_{S}(x) \leq \varepsilon\right\}, \\
M_{2}(\varepsilon)=\left\{x \in K: f(x, y)+\varepsilon \geq 0, \forall y \in S, d_{D}(g(x)) \leq \varepsilon\right\} .
\end{gathered}
$$

Theorem 3.1. Let $(X,\|\cdot\|)$ be a Banach space. Then, $(E P)$ is type I LP well-posed if and only if the solution set $\Gamma$ of $(E P)$ is nonempty, compact, and

$$
e\left(M_{1}(\varepsilon), \Gamma\right) \longrightarrow 0 \text { as } \varepsilon \longrightarrow 0 .
$$

Proof. Let (EP) be type I LP well-posed. Then $\Gamma$ is nonempty and compact. Now, we prove that (3.3) holds. Suppose to the contrary that there exist $\gamma>0,\left\{\varepsilon_{n}\right\}$ with $\varepsilon_{n} \rightarrow 0$, and $x_{n} \in M_{1}\left(\varepsilon_{n}\right)$ such that

$$
d_{\Gamma}\left(x_{n}\right) \geq \gamma
$$

Since $\left\{x_{n}\right\} \subset M_{1}\left(\varepsilon_{n}\right)$, we know that $\left\{x_{n}\right\}$ is a type I LP approximating solution sequence for (EP). By the type I LP well-posedness of (EP), there exists a subsequence $\left\{x_{n_{k}}\right\}$ of $\left\{x_{n}\right\}$ converging to some point of $\Gamma$. This contradicts (3.4) and so (3.3) holds. 
Conversely, suppose that $\Gamma$ is nonempty, compact, and (3.3) holds. Let $\left\{x_{n}\right\}$ be a type I LP approximating solution sequence for (EP). Then there exists a sequence $\left\{\varepsilon_{n}\right\}$ with $\varepsilon_{n}>0$ and $\varepsilon_{n} \rightarrow 0$ such that $f\left(x_{n}, y\right)+\varepsilon_{n} \geq 0$ for all $y \in S$ and $d_{S}\left(x_{n}\right) \leq \varepsilon_{n}$. Thus, $\left\{x_{n}\right\} \subset M_{1}\left(\varepsilon_{n}\right)$. It follows from (3.3) that there exists a sequence $\left\{z_{n}\right\} \subset \Gamma$ such that

$$
\left\|x_{n}-z_{n}\right\|=d\left(x_{n}, \Gamma\right) \leq e\left(M_{1}\left(\varepsilon_{n}\right), \Gamma\right) \longrightarrow 0 .
$$

Since $\Gamma$ is compact, there exists a subsequence $\left\{z_{n_{k}}\right\}$ of $\left\{z_{n}\right\}$ converging to $x_{0} \in \Gamma$, and so the corresponding subsequence $\left\{x_{n_{k}}\right\}$ of $\left\{x_{n}\right\}$ converges to $x_{0}$. Therefore, (EP) is type I LP wellposed. This completes the proof.

Example 3.2. Let $X=Y=R, K=[0,2]$, and $D=[0,1]$. Let

$$
g(x)=x, \quad f(x, y)=(x-y)^{2}, \quad \forall x, y \in X
$$

Then it is easy to compute that $S=[0,1], \Gamma=[0,1]$, and $M_{1}(\varepsilon)=[0,1+\varepsilon]$. It follows that $e\left(M_{1}(\varepsilon), \Gamma\right) \rightarrow 0$ as $\varepsilon \rightarrow 0$. By Theorem 3.1, (EP) is type I LP well-posed.

The following example illustrates that the compactness condition in Theorem 3.1 is essential.

Example 3.3. Let $X=Y=R, K=[0,+\infty), D=[0,+\infty)$, and let $g$ and $f$ be the same as in Example 3.2. Then, it is easy to compute that $S=[0,+\infty), \Gamma=[0,+\infty), M_{1}(\varepsilon)=[0,+\infty)$, and $e\left(M_{1}(\varepsilon), \Gamma\right) \rightarrow 0$ as $\varepsilon \rightarrow 0$. Let $x_{n}=n$ for $n=1,2, \ldots$ Then, $\left\{x_{n}\right\}$ is an approximating solution sequence for (EP), which has no convergent subsequence. This implies that (EP) is not type I LP well-posed.

Furi and Vignoli [8] characterized well-posedness of the optimization problem (defined in a complete metric space $\left.\left(S, d_{1}\right)\right)$ by the use of the Kuratowski measure of noncompactness of a subset $A$ of $X$ defined as

$$
\mu(A)=\inf \left\{\varepsilon>0: A \subseteq \bigcup_{i=1}^{n} A_{i}, \operatorname{diam} A_{i}<\varepsilon, i=1,2, \ldots, n\right\}
$$

where diam $A_{i}$ is the diameter of $A_{i}$ defined by diam $A_{i}=\sup \left\{d_{1}\left(x_{1}, x_{2}\right): x_{1}, x_{2} \in A_{i}\right\}$.

Now, we give a Furi-Vignoli-type characterization for the various LP well-posed.

Theorem 3.4. Let $(X,\|\cdot\|)$ be a Banach space and $\Gamma \neq \varnothing$. Assume that $f$ is upper semicontinuous on $K \times K$ with respect to the first argument. Then, $(E P)$ is type I LP well-posed if and only if

$$
\lim _{\varepsilon \rightarrow 0} \mu\left(M_{1}(\varepsilon)\right)=0
$$


Proof. Let (EP) be type I LP well-posed. It is obvious that $\Gamma$ is nonempty and compact. As proved in Theorem 3.1, $e\left(M_{1}(\varepsilon), \Gamma\right) \rightarrow 0$ as $\varepsilon \rightarrow 0$. Since $\Gamma$ is compact, $\mu(\Gamma)=0$ and the following relation holds (see, e.g., [7]):

$$
\mu\left(M_{1}(\varepsilon)\right) \leq 2 H\left(M_{1}(\varepsilon), \Gamma\right)+\mu(\Gamma)=2 H\left(M_{1}(\varepsilon), \Gamma\right)=2 e\left(M_{1}(\varepsilon), \Gamma\right) .
$$

Therefore, (3.8) holds.

In order to prove the converse, suppose that (3.8) holds. We first show that $M_{1}(\varepsilon)$ is nonempty and closed for any $\varepsilon>0$. In fact, the nonemptiness of $M_{1}(\varepsilon)$ follows from the fact that $\Gamma \neq \varnothing$. Let $\left\{x_{n}\right\} \subset M_{1}(\varepsilon)$ with $x_{n} \rightarrow x_{0}$. Then

$$
\begin{gathered}
d_{S}\left(x_{n}\right) \leq \varepsilon \\
f\left(x_{n}, y\right)+\varepsilon \geq 0, \quad \forall y \in S .
\end{gathered}
$$

It follows from (3.10) that

$$
d_{S}\left(x_{0}\right) \leq \varepsilon
$$

By the upper semicontinuity of $f$ with respect to the first argument and (3.11), we have $f\left(x_{0}, y\right)+\varepsilon \geq 0$ for all $y \in S$, which together with (3.12) yields $x_{0} \in M_{1}(\varepsilon)$, and so $M_{1}(\varepsilon)$ is closed. Now we prove that $\Gamma$ is nonempty and compact. Observe that $\Gamma=\bigcap_{\varepsilon>0} M_{1}(\varepsilon)$. Since $\lim _{\varepsilon \rightarrow 0} \mu\left(M_{1}(\varepsilon)\right)=0$, by the Kuratowski theorem ([37], [38, page 318]), we have

$$
H\left(M_{1}(\varepsilon), \Gamma\right) \longrightarrow 0 \text { as } \varepsilon \longrightarrow 0
$$

and so $\Gamma$ is nonempty and compact.

Let $\left\{x_{n}\right\}$ be a type I LP approximating solution sequence for (EP). Then, there exists a sequence $\left\{\varepsilon_{n}\right\}$ with $\varepsilon_{n}>0$ and $\varepsilon_{n} \rightarrow 0$ such that $f\left(x_{n}, y\right)+\varepsilon_{n} \geq 0$ for all $y \in S$ and $d_{S}\left(x_{n}\right) \leq \varepsilon_{n}$. Thus, $\left\{x_{n}\right\} \subset M_{1}\left(\varepsilon_{n}\right)$. This fact together with (3.13) shows that $d_{\Gamma}\left(x_{n}\right) \rightarrow 0$. By Lemma 2.11, $(\mathrm{EP})$ is type I LP well-posed. This completes the proof.

In the similar way to Theorems 3.1 and 3.4, we can prove the following Theorems 3.5 and 3.6, respectively.

Theorem 3.5. Let $(X,\|\cdot\|)$ be a Banach space. Then, $(E P)$ is generalized type I LP well-posed if and only if the solution set $\Gamma$ of $(E P)$ is nonempty, compact, and $e\left(M_{2}(\varepsilon), \Gamma\right) \rightarrow 0$ as $\varepsilon \rightarrow 0$.

Theorem 3.6. Let $(X,\|\cdot\|)$ be a Banach space and $\Gamma \neq \varnothing$. Assume that $f$ is upper semicontinuous on $K \times K$ with respect to the first argument. Then, $(E P)$ is generalized type I LP well-posed if and only if $\lim _{\varepsilon \rightarrow 0} \mu\left(M_{2}(\varepsilon)\right)=0$.

In the following we consider a real-valued function $c=c(t, s)$ defined for $s, t \geq 0$ sufficiently small, such that

$$
\begin{gathered}
c(t, s) \geq 0, \quad \forall t, s, \quad c(0,0)=0, \\
s_{n} \longrightarrow 0, \quad t_{n} \geq 0, \quad c\left(t_{n}, s_{n}\right) \longrightarrow 0, \quad \text { imply } t_{n} \longrightarrow 0 .
\end{gathered}
$$

By using [33, Theorem 2.1] and Lemma 2.10, we have the following theorem. 
Theorem 3.7. Let (EP) be type II LP well-posed. Then there exists a function c satisfying (3.14) such that

$$
|h(x)| \geq c\left(d_{\Gamma}(x), d_{S}(x)\right), \quad \forall x \in K,
$$

where $h(x)$ is defined by (2.11). Conversely, suppose that $\Gamma$ is nonempty and compact, and (3.15) holds for some c satisfying (3.14). Then, (EP) is type II LP well-posed.

Similarly, we have the next theorem by applying [33, Theorem 2.2] and Lemma 2.10.

Theorem 3.8. Let (EP) be generalized type II LP well-posed. Then there exists a function c satisfying (3.14) such that

$$
|h(x)| \geq c\left(d_{\Gamma}(x), d_{D}(g(x))\right), \quad \forall x \in K,
$$

where $h(x)$ is defined by (2.11). Conversely, suppose that $\Gamma$ is nonempty and compact, and (3.16) holds for some c satisfying (3.14). Then, (EP) is generalized type II LP well-posed.

\section{Sufficient conditions of LP well-posedness for (EP)}

In this section, we derive several sufficient conditions for various types of LP well-posedness for $(\mathrm{EP})$.

Definition 4.1. Let $Z$ be a topological space and let $Z_{1} \subset Z$ be a nonempty subset. Suppose that $G: Z \rightarrow R \cup\{+\infty\}$ is an extended real-valued function. The function $G$ is said to be levelcompact on $Z_{1}$ if, for any $s \in R$, the subset $\left\{z \in Z_{1}: G(z) \leq s\right\}$ is compact.

Proposition 4.2. Suppose that $f$ is upper semicontinuous on $K \times K$ with respect to the first argument and $\Gamma \neq \varnothing$. Then, $(E P)$ is type I LP well-posed if one of the following conditions holds:

(i) there exists $\delta_{1}>0$ such that $S\left(\delta_{1}\right)$ is compact, where

$$
S\left(\delta_{1}\right)=\left\{x \in K: d_{S}(x) \leq \delta_{1}\right\}
$$

(ii) the function $h$ defined by (2.11) is level-compact on $K$;

(iii) $X$ is a finite-dimensional normed space and

$$
\lim _{x \in K,\|x\| \rightarrow+\infty} \max \left\{h(x), d_{S}(x)\right\}=+\infty ;
$$

(iv) there exists $\delta_{1}>0$ such that $h$ is level-compact on $S\left(\delta_{1}\right)$ defined by (4.1).

Proof. (i) Let $\left\{x_{n}\right\}$ be a type I LP approximating solution sequence for (EP). Then, there exists a sequence $\left\{\varepsilon_{n}\right\}$ with $\varepsilon_{n}>0$ and $\varepsilon_{n} \rightarrow 0$ such that

$$
\begin{gathered}
d_{S}\left(x_{n}\right) \leq \varepsilon_{n} \\
f\left(x_{n}, y\right)+\varepsilon_{n} \geq 0, \quad \forall y \in S .
\end{gathered}
$$


From (4.3), without loss of generality, we can assume that $\left\{x_{n}\right\} \subset S\left(\delta_{1}\right)$. Since $S\left(\delta_{1}\right)$ is compact, there exists a subsequence $\left\{x_{n_{j}}\right\}$ of $\left\{x_{n}\right\}$ and $x_{0} \in S\left(\delta_{1}\right)$ such that $x_{n_{j}} \rightarrow x_{0}$. This fact combined with (4.3) yields $x_{0} \in S$. Furthermore, it follows from (4.4) that $f\left(x_{n_{j}}, y\right) \geq-\varepsilon_{n_{j}}$ for all $y \in S$. By the upper semicontinuity of $f$ with respect to the first argument, we have $f\left(x_{0}, y\right) \geq 0$ for all $y \in S$ and so $x_{0} \in \Gamma$. Thus, (EP) is type I LP well-posed.

It is easy to see that condition (ii) implies condition (iv). Now, we show that condition (iii) implies condition (iv). Since $X$ is a finite-dimensional space and the function $h$ is lower semicontinuous on $S\left(\delta_{1}\right)$, we need only to prove that, for any $s \in R$ and $\delta_{1}>0$, the set $B=\{x \in$ $\left.S\left(\delta_{1}\right): h(x) \leq s\right\}$ is bounded, and thus $B$ is closed. Suppose by contradiction that there exist $s \in R$ and $\left\{x_{n}\right\} \subset S\left(\delta_{1}\right)$ such that $\|x\| \rightarrow+\infty$ and $h\left(x_{n}\right) \leq s$. It follows from $\left\{x_{n}\right\} \subset S\left(\delta_{1}\right)$ that $d_{S}\left(x_{n}\right) \leq \delta_{1}$ and so

$$
\max \left\{h\left(x_{n}\right), d_{S}\left(x_{n}\right)\right\} \leq \max \left\{s, \delta_{1}\right\}
$$

which contradicts (4.2).

Therefore, we need only to prove that if condition (iv) holds, then (EP) is type I LP wellposed. Suppose that condition (iv) holds. From (4.3), without loss of generality, we can assume that $\left\{x_{n}\right\} \subset S\left(\delta_{1}\right)$. By (4.4), we can assume without loss of generality that $\left\{x_{n}\right\} \subset\{x \in K$ : $h(x) \leq m\}$ for some $m>0$. Since $h$ is level-compact on $S\left(\delta_{1}\right)$, the subset $\left\{x \in S\left(\delta_{1}\right): h(x) \leq m\right\}$ is compact. It follows that there exist a subsequence $\left\{x_{n_{j}}\right\}$ of $\left\{x_{n}\right\}$ and $x_{0} \in S\left(\delta_{1}\right)$ such that $x_{n_{j}} \rightarrow x_{0}$. This together with (4.3) yields $x_{0} \in S$. Furthermore, by the upper semicontinuity of $f$ with respect to the first argument and (4.4), we obtain $x_{0} \in \Gamma$. This completes the proof.

Similarly, we can prove the next proposition.

Proposition 4.3. Assume that $f$ is upper semicontinuous on $K \times K$ with respect to the first argument and $\Gamma \neq \varnothing$. Then, $(E P)$ is generalized type I LP well-posed if one of the following conditions holds:

(i) there exists $\delta_{1}>0$ such that $S_{1}\left(\delta_{1}\right)$ is compact, where

$$
S_{1}\left(\delta_{1}\right)=\left\{x \in K: d_{D}(g(x)) \leq \delta_{1}\right\}
$$

(ii) the function h defined by (2.11) is level-compact on $K$;

(iii) $X$ is a finite-dimensional normed space and

$$
\lim _{x \in K,\|x\| \rightarrow+\infty} \max \left\{h(x), d_{D}(g(x))\right\}=+\infty ;
$$

(iv) there exists $\delta_{1}>0$ such that $h$ is level-compact on $S_{1}\left(\delta_{1}\right)$ defined by (4.6).

Proposition 4.4. Let $X$ be a finite-dimensional space, $f$ an upper semicontinuous function on $K \times K$ with respect to the first argument, and $\Gamma \neq \varnothing$. Suppose that there exists $y_{0} \in S$ such that

$$
\lim _{\|x\| \rightarrow+\infty}-f\left(x, y_{0}\right)=+\infty
$$

Then, (EP) is type I LP well-posed. 
Proof. Let $\left\{x_{n}\right\}$ be a type I LP approximating solution sequence for (EP). Then, there exists a sequence $\left\{\varepsilon_{n}\right\}$ with $\varepsilon_{n}>0$ and $\varepsilon_{n} \rightarrow 0$ such that

$$
\begin{gathered}
d_{S}\left(x_{n}\right) \leq \varepsilon_{n} \\
f\left(x_{n}, y\right)+\varepsilon_{n} \geq 0, \quad \forall y \in S .
\end{gathered}
$$

By (4.9), without loss of generality, we can assume that $\left\{x_{n}\right\} \subset S\left(\delta_{1}\right)$, where $S\left(\delta_{1}\right)$ is defined by (4.1) with some $\delta_{1}>0$. Now, we claim that $\left\{x_{n}\right\}$ is bounded. Indeed, if $\left\{x_{n}\right\}$ is unbounded, without loss of generality, we can suppose that $\left\|x_{n}\right\| \rightarrow+\infty$. By (4.8), we obtain $\lim _{n \rightarrow+\infty}-$ $f\left(x_{n}, y_{0}\right)=+\infty$, which contradicts (4.10) when $n$ is sufficiently large. Therefore, we can assume without loss of generality that $x_{n} \rightarrow x_{0} \in K$. This fact together with (4.9) yields $x_{0} \in S$. By the upper semicontinuity of $f$ with respect to the first argument and (4.10), we get $x_{0} \in \Gamma$. This completes the proof.

Example 4.5. Let $X=Y=R, K=[0,2]$, and $D=[0,1]$. Let

$$
g(x)=\frac{1}{2} x, \quad f(x, y)=y(y-x), \quad \forall x, y \in X
$$

Then it is easy to see that $S=[0,2]$ and condition (4.8) in Proposition 4.4 is satisfied.

In view of the generalized type I LP well-posedness, we can similarly prove the following proposition.

Proposition 4.6. Let $X$ be a finite-dimensional space, $f$ an upper semicontinuous function on $K \times K$ with respect to the first argument, and $\Gamma \neq \varnothing$. If there exists $y_{0} \in S$ such that $\lim _{\|x\| \rightarrow+\infty}-f\left(x, y_{0}\right)=$ $+\infty$, then $(E P)$ is generalized type I LP well-posed.

Now, we consider the case when $Y$ is a normed space, $D$ is a closed and convex cone with nonempty interior int $D$. Let $e \in \operatorname{int} D$. For any $\delta \geq 0$, denote

$$
S_{2}(\delta)=\{x \in K: g(x) \in D-\delta e\}
$$

Proposition 4.7. Let $Y$ be a normal space, let $D$ be a closed convex cone with nonempty interior int $D$ and $e \in \operatorname{int} D$. Assume that $f$ is upper semicontinuous on $K \times K$ with respect to the first argument and $\Gamma \neq \varnothing$. If there exists $\delta_{1}>0$ such that the function $h(x)$ defined by $(2.11)$ is level-compact on $S_{2}\left(\delta_{1}\right)$, then (EP) is generalized type I LP well-posed.

Proof. Let $\left\{x_{n}\right\}$ be a generalized type I LP approximating solution sequence for (EP). Then, there exists a sequence $\left\{\varepsilon_{n}\right\}$ with $\varepsilon_{n}>0$ and $\varepsilon_{n} \rightarrow 0$ such that

$$
\begin{gathered}
d_{D}\left(g\left(x_{n}\right)\right) \leq \varepsilon_{n}, \\
f\left(x_{n}, y\right)+\varepsilon_{n} \geq 0, \quad \forall y \in S .
\end{gathered}
$$

It follows from (4.13) that there exists $\left\{s_{n}\right\} \subset D$ such that $\left\|g\left(x_{n}\right)-s_{n}\right\| \leq 2 \varepsilon_{n}$ and so

$$
g\left(x_{n}\right)-s_{n} \in 2 \varepsilon_{n} B
$$


where $B$ is a closed unit ball of $Y$. Now, we show that there exists $M_{0}>0$ such that

$$
B \subset D-M_{0} e
$$

Suppose by contradiction that there exist $b_{n} \in B$ and $0<M_{n} \rightarrow+\infty$ such that $b_{n}+M_{n} e \notin D$ for $n=1,2, \ldots$ Then $b_{n}+M_{n} e \notin \operatorname{int} D$ and so

$$
\frac{b_{n}}{M_{n}}+e \notin \operatorname{int} D, \quad n=1,2, \ldots
$$

Taking the limit in (4.17), we obtain $e \notin$ int $D$. This gives a contradiction to the assumption. Thus, the combination of (4.15) and (4.16) yields $g\left(x_{n}\right)-s_{n} \in D-2 M_{0} \varepsilon_{n} e$ and so $g\left(x_{n}\right) \in$ $D-2 M_{0} \varepsilon_{n} e$. By (4.12), we can assume without loss of generality that

$$
x_{n} \in S_{2}\left(\delta_{1}\right)
$$

for $2 M_{0} \varepsilon_{n} \rightarrow 0$ as $n \rightarrow+\infty$. It follows from (4.14) that

$$
h\left(x_{n}\right) \leq \varepsilon_{n}, \quad n=1,2, \ldots
$$

By (4.18), (4.19), and the level-compactness of $h$ on $S_{2}\left(\delta_{1}\right)$, we know that there exist a subsequence $\left\{x_{n_{j}}\right\}$ of $\left\{x_{n}\right\}$ and $x_{0} \in S_{2}\left(\delta_{1}\right)$ such that $x_{n_{j}} \rightarrow x_{0}$. Taking the limit in (4.13) (with $n$ replaced by $\left.n_{j}\right)$, we obtain $x_{0} \in S$. Furthermore, we get $f\left(x_{0}, y\right) \geq 0$ for all $y \in S$. Therefore, $x_{0} \in \Gamma$. This completes the proof.

\section{Relations among various type of LP well-posedness for (EP)}

In this section, we will investigate further relationships among the various types of LP wellposedness for (EP).

By definition, it is easy to see that the following result holds.

Theorem 5.1. Assume that there exist $\delta_{1}, \alpha>0$ and $c>0$ such that

$$
d_{S}(x) \leq c d_{D}^{\alpha}(g(x)), \quad \forall x \in S_{1}\left(\delta_{1}\right)
$$

where $S_{1}\left(\delta_{1}\right)$ is defined by (4.6). If (EP) is type I (type II) LP well-posed, then (EP) is generalized type I (type II) LP well-posed.

Definition 5.2 (see [6]). Let $W$ be a topological space. A set-valued mapping $F: W \rightarrow 2^{X}$ is said to be upper Hausdorff semicontinuous at $w \in W$ if, for any $\varepsilon>0$, there exists a neighborhood $U$ of $w$ such that $F(U) \subset B(F(w), \varepsilon)$, where, for $Z \subset X$ and $r>0, B(Z, r)=\left\{x \in X: d_{Z}(x) \leq r\right\}$.

Clearly, $S_{1}\left(\delta_{1}\right)$ given by (4.6) is a set-valued mapping from $R_{+}$to $X$.

Theorem 5.3. Suppose that the set-valued mapping $S_{1}\left(\delta_{1}\right)$ defined by (4.6) is upper Hausdorff semicontinuous at $0 \in R_{+}$. If (EP) is type I (type II) LP well-posed, then (EP) is generalized type I (type II) LP well-posed. 
Proof. We prove only type I case, the other case can be proved similarly. Let $\left\{x_{n}\right\}$ be a generalized type I LP approximating solution sequence for (EP). Then there exists a sequence $\varepsilon_{n} \rightarrow 0$ such that

$$
\begin{gathered}
d_{D}\left(g\left(x_{n}\right)\right) \leq \varepsilon_{n} \\
f\left(x_{n}, y\right)+\varepsilon_{n} \geq 0, \quad \forall y \in S .
\end{gathered}
$$

Note that $S_{1}\left(\delta_{1}\right)$ is upper Hausdorff semicontinuous at 0 . This fact together with (5.2) yields that $d_{S}\left(x_{n}\right) \leq \varepsilon_{n}$, which combining (5.3) implies that $\left\{x_{n}\right\}$ is type I LP approximating solution sequence. Since (EP) is type I LP well-posed, there exists a subsequence $\left\{x_{n_{j}}\right\}$ of $\left\{x_{n}\right\}$ converging to some point of $\Gamma$. Therefore, $(\mathrm{EP})$ is generalized type I LP well-posed. This completes the proof.

Let $Y$ be a normed space and set

$$
S_{3}(y)=\{x \in K: g(x) \in D+y\}, \quad \forall y \in Y .
$$

Clearly, $S_{3}(y)$ is a set-valued mapping from $Y$ to $X$. Similar to the proof of Theorem 5.3, we can prove the following result.

Theorem 5.4. Assume that the set-valued mapping $S_{3}(y)$ defined by (5.4) is upper Hausdorff semicontinuous at $0 \in Y$. If (EP) is type I (type II) LP well-posed, then (EP) is generalized type I (type II) LP well-posed.

Corollary 5.5. Let $D$ be a closed and convex cone with nonempty interior int $D$ and $e \in$ int $D$. Suppose that the set-valued mapping $S_{2}(\delta)$ defined by (4.12) is upper Hausdorff semicontinuous at $0 \in R_{+}$. If $(E P)$ is type I (type II) LP well-posed, then (EP) is generalized type I (type II) LP well-posed.

\section{Acknowledgments}

The work of the second author was supported by the National Natural Science Foundation of China (10671135), the Specialized Research Fund for the Doctoral Program of Higher Education (20060610005), and the open fund (PLN0703) of State Key Laboratory of Oil and Gas Reservoir Geology and Exploitation (Southwest Petroleum University), and the work of the third author was supported by the ARC grant from the Australian Research Council. The authors are grateful to Professor Simeon Reich and the referees for their valuable comments and suggestions.

\section{References}

[1] E. Blum and W. Oettli, "From optimization and variational inequalities to equilibrium problems," The Mathematics Student, vol. 63, no. 1-4, pp. 123-145, 1994.

[2] G.-Y. Chen, X. X. Huang, and X. Q. Yang, Vector Optimization: Set-Valued and Variational Analysis, vol. 541 of Lecture Notes in Economics and Mathematical Systems, Springer, Berlin, Germany, 2005.

[3] F. Giannessi, Ed., Vector Variational Inequalities and Vector Equilibria: Mathematical Theories, vol. 38 of Nonconvex Optimization and Its Applications, Kluwer Academic, Dordrecht, The Netherlands, 2000.

[4] A. N. Tykhonov, "On the stability of the functional optimization problems," USSR Computational Mathematics and Mathematical Physics, vol. 6, no. 4, pp. 28-33, 1966. 
[5] E. S. Levitin and B. T. Polyak, "Convergence of minimizing sequences in conditional extremum problem," Soviet Mathematics Doklady, vol. 7, pp. 764-767, 1966.

[6] E. Bednarczuk and J.-P. Penot, "Metrically well-set minimization problems," Applied Mathematics and Optimization, vol. 26, no. 3, pp. 273-285, 1992.

[7] A. L. Dontchev and T. Zolezzi, Well-Posed Optimization Problems, vol. 1543 of Lecture Notes in Mathematics, Springer, Berlin, Germany, 1993.

[8] M. Furi and A. Vignoli, "About well-posed minimization problems for functionals in metric spaces," Journal of Optimization Theory and Appllications, vol. 5, no. 3, pp. 225-229, 1970.

[9] R. Lucchetti and F. Patrone, "Hadamard and Tyhonov well-posedness of a certain class of convex functions," Journal of Mathematical Analysis and Applications, vol. 88, no. 1, pp. 204-215, 1982.

[10] J. P. Revalski, "Hadamard and strong well-posedness for convex programs," SIAM Journal on Optimization, vol. 7, no. 2, pp. 519-526, 1997.

[11] T. Zolezzi, "Well-posedness criteria in optimization with application to the calculus of variations," Nonlinear Analysis: Theory, Methods \& Applications, vol. 25, no. 5, pp. 437-453, 1995.

[12] T. Zolezzi, "Extended well-posedness of optimization problems," Journal of Optimization Theory and Applications, vol. 91, no. 1, pp. 257-266, 1996.

[13] T. Zolezzi, "Well-posedness and optimization under perturbations," Annals of Operations Research, vol. 101, no. 1-4, pp. 351-361, 2001.

[14] S. Reich and A. J. Zaslavski, "Well-posedness of generalized best approximation problems," Nonlinear Functional Analysis and Applications, vol. 7, no. 1, pp. 115-128, 2002.

[15] S. Reich and A. J. Zaslavski, "Porous sets and generalized best approximation problems," Nonlinear Analysis Forum, vol. 9, no. 2, pp. 135-152, 2004.

[16] S. Reich and A. J. Zaslavski, "Well-posedness and porosity in best approximation problems," Topological Methods in Nonlinear Analysis, vol. 18, no. 2, pp. 395-408, 2001.

[17] E. Bednarczuk, "Well-posedness of vector optimization problems," in Recent Advances and Historical Development of Vector Optimization Problems, J. Jahn and W. Krabs, Eds., vol. 294 of Lecture Notes in Economics and Mathematical Systems, pp. 51-61, Springer, Berlin, Germany, 1987.

[18] E. Bednarczuk, "An approach to well-posedness in vector optimization: consequences to stability," Control and Cybernetics, vol. 23, no. 1-2, pp. 107-122, 1994.

[19] G. P. Crespi, A. Guerraggio, and M. Rocca, "Well-posedness in vector optimization problems and vector variational inequalities," Journal of Optimization Theory and Applications, vol. 132, no. 1, pp. 213226, 2007.

[20] X. X. Huang, "Extended well-posedness properties of vector optimization problems," Journal of Optimization Theory and Applications, vol. 106, no. 1, pp. 165-182, 2000.

[21] P. Loridan, "Well-posedness in vector optimization," in Recent Developments in Well-Posed Variational Problems, vol. 331 of Mathematics and Its Applications, pp. 171-192, Kluwer Academic, Dordrecht, The Netherlands, 1995.

[22] E. Miglierina and E. Molho, "Well-posedness and convexity in vector optimization," Mathematical Methods of Operations Research, vol. 58, no. 3, pp. 375-385, 2003.

[23] E. Miglierina, E. Molho, and M. Rocca, "Well-posedness and scalarization in vector optimization," Journal of Optimization Theory and Applications, vol. 126, no. 2, pp. 391-409, 2005.

[24] A. J. Zaslavski, "Generic well-posedness of optimal control problems without convexity assumptions," SIAM Journal on Control and Optimization, vol. 39, no. 1, pp. 250-280, 2000.

[25] A. J. Zaslavski, "Generic well-posedness of nonconvex constrained variational problems," Journal of Optimization Theory and Applications, vol. 130, no. 3, pp. 527-543, 2006.

[26] M. B. Lignola, "Well-posedness and L-well-posedness for quasivariational inequalities," Journal of Optimization Theory and Applications, vol. 128, no. 1, pp. 119-138, 2006.

[27] M. B. Lignola and J. Morgan, "Well-posedness for optimization problems with constraints defined by variational inequalities having a unique solution," Journal of Global Optimization, vol. 16, no. 1, pp. 57-67, 2000.

[28] M. B. Lignola and J. Morgan, " $\alpha$-well-posedness for Nash equilibria and for optimization problems with Nash equilibrium constraints," Journal of Global Optimization, vol. 36, no. 3, pp. 439-459, 2006.

[29] M. Margiocco, F. Patrone, and L. Pusillo Chicco, "A new approach to Tikhonov well-posedness for Nash equilibria," Optimization, vol. 40, no. 4, pp. 385-400, 1997.

[30] M. Margiocco, F. Patrone, and L. Pusillo Chicco, "Metric characterizations of Tikhonov well-posedness in value," Journal of Optimization Theory and Applications, vol. 100, no. 2, pp. 377-387, 1999. 
[31] J. Yu, H. Yang, and C. Yu, "Well-posed Ky Fan's point, quasi-variational inequality and Nash equilibrium problems," Nonlinear Analysis: Theory, Methods \& Applications, vol. 66, no. 4, pp. 777-790, 2007.

[32] A. S. Konsulova and J. P. Revalski, "Constrained convex optimization problems-well-posedness and stability," Numerical Functional Analysis and Optimization, vol. 15, no. 7\&8, pp. 889-907, 1994.

[33] X. X. Huang and X. Q. Yang, "Generalized Levitin-Polyak well-posedness in constrained optimization," SIAM Journal on Optimization, vol. 17, no. 1, pp. 243-258, 2006.

[34] X. X. Huang and X. Q. Yang, "Levitin-Polyak well-posedness of constrained vector optimization problems," Journal of Global Optimization, vol. 37, no. 2, pp. 287-304, 2007.

[35] X. X. Huang and X. Q. Yang, "Levitin-Polyak well-posedness in generalized variational inequality problems with functional constraints," Journal of Industral Management and Optimizations, vol. 3, no. 4, pp. 671-684, 2007.

[36] G. Mastroeni, "Gap functions for equilibrium problems," Journal of Global Optimization, vol. 27, no. 4, pp. 411-426, 2003.

[37] K. Kuratowski, Topology, vol. 1, Academic Press, New York, NY, USA, 1968.

[38] K. Kuratowski, Topology, vol. 2, Academic Press, New York, NY, USA, 1968. 